\title{
Rapid urease test provides specific identification of Campylobacter pylori in antral mucosal biopsies
}

\author{
M.-L. Szeto, ${ }^{1}$ R.E. Pounder, ${ }^{1}$ S.J. Hamilton-Dutoit ${ }^{2}$ and A.P. Dhillon ${ }^{2}$ \\ Academic Departments of ${ }^{1}$ Medicine and ${ }^{2}$ Histopathology, Royal Free Hospital School of Medicine, \\ Rowland Hill Street, London NW3 2PF, UK.
}

\begin{abstract}
Summary: In a prospective study, three antral biopsies were taken from 175 dyspeptic patients during routine endoscopy. One biopsy was inserted immediately into a gel-containing well of a CLOtest slide and two biopsies were sent to histopathology. Using the CLOtest, 84 of the 175 samples (48\%) detected urease activity in the gastric biopsy, suggesting infection with Campylobacter pylori. Histopathological examination by two independent observers reported that 93 $(52 \%)$ of the biopsies contained Campylobacter-like organisms. The CLOtest was found to have a specificity of 1.0 and sensitivity of 0.91 , providing a rapid identification of most patients harbouring the organism in their gastric mucus.
\end{abstract}

\section{Introduction}

Campylobacter pylori is found in the mucus of the gastric antrum of some patients with peptic ulcer disease, always in association with chronic gastritis. $^{1-3}$ It is possible that $C$. pylori and chronic gastritis have a role in the initiation or persistence of peptic ulceration; C. pylori may be an important factor in some types of non-ulcer dyspepsia. Chronic gastritis cannot be diagnosed visually at endoscopy, but the organism can be identified, in due course, in endoscopic gastric mucosal biopsies by microbiological culture or histological examination. However, C. pylori produces abundant urease - an enzyme which can be detected by the rapid urease slide CLOtest. ${ }^{4}$ The CLOtest is a plastic slide with a well which contains a gel pellet; the gel contains urea, phenyl red and a bacteriostatic agent. The endoscopic mucosal biopsy is placed in the gel (Figure 1) and, if the biopsy contains urease activity, urea rapidly releases ammonia which is detected by the phenyl red indicator. The test detects preformed urease activity in the biopsy - it does not depend upon proliferation or viability of C. pylori in the gastric mucus. The present prospective study compared the results of the CLOtest with routine and 'review' histological reporting of antral mucosal biopsies.

Correspondence: R.E. Pounder, M.A., M.D., F.R.C.P. Accepted: 3 August 1988

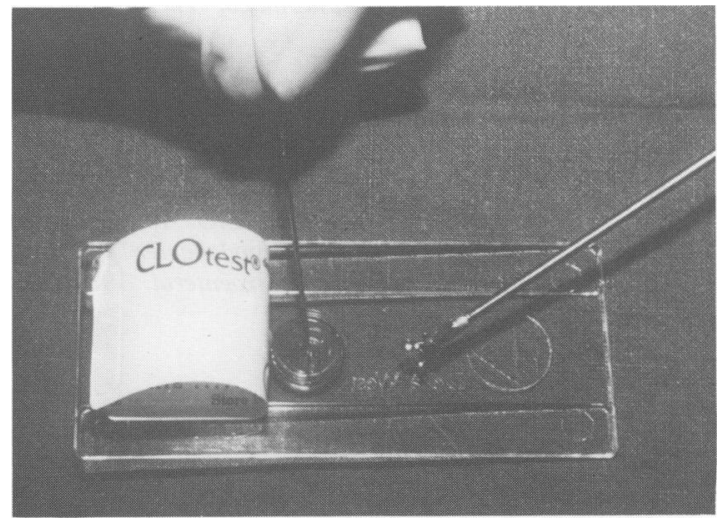

Figure 1 A needle is used to insert an endoscopic mucosal biopsy into the gel pellet of a CLOtest slide. The well, containing the gel pellet, is exposed by peeling back a paper label and resealed for incubation.

Patients, methods and results

Three antral biopsies were taken from 175 dyspeptic patients during routine endoscopy at the Royal Free Hospital, London. One biopsy was inserted immediately into the gel pellet of the CLOtest slide, and two biopsies were sent to histopathology. The

(C) The Fellowship of Postgraduate Medicine, 1988 
slide was kept warm in the endoscopist's shirt pocket and reviewed regularly for 24 hours; the time of any colour change was noted. Histology slides were stained with haematoxylin and eosin, reported by the routine histological service of the hospital, and then 'reviewed' independently by two histopathologists (all reporting was without the knowledge of the CLOtest result).

Eighty-four $(48 \%)$ of the 175 samples had positive red colouration with the CLOtest (of which 66, 74 and $92 \%$ were positive at 20,60 and 180 minutes, respectively). Routine histology reported that $79(45 \%)$ of the biopsies contained Campylobacter-like organisms, but 'review histopathology' reported that $93(52 \%)$ of the biopsies were positive. All of the CLOtest positive samples were reported at review to contain Campylobacterlike organisms (specificity 1.0), but nine of the 93 'review' positive samples were negative by CLOtest (sensitivity 0.91 ). The CLOtest did not give a single false positive result.

\section{References}

1. Tytgat, G.N.J. \& Rauws, E.A.J. Significance of Campylobacter pylori. Aliment Pharmacol Therap 1987, 1 (suppl 1): 527S-539S.

2. Dooley, C.P. \& Cohen, H. The clinical significance of Campylobacter pylori. Ann Int Med 1988, 108: 70-79.

3. Bartlett, J.E. Campylobacter pylori: fact or fancy? Gastroenterology 1988, 94: 229-238.

4. Marshall, B.J., Warren, J.R., Francis, G.J., Langton, S.R., Goodwin, C.S. \& Blincow, E.D. Rapid urease test in the management of Campylobacter pyloridisassociated gastritis. Am J Gastroenterol 1987, 82: 200-210.

\section{Discussion}

The CLOtest provides a rapid, highly specific and reasonably sensitive test for $C$. pylori in the antral mucosa, confirming the results of two smaller series. $^{4,5}$ Although the exact role of $C$. pylori in either peptic ulceration or non-ulcer dyspepsia remains controversial ${ }^{1-3}$ this technique can identify most patients harbouring the organism in their gastric mucus whilst they are still in hospital recovering from their diagnostic endoscopy, allowing an immediate decision concerning appropriate therapy for dyspepsia. ${ }^{1}$

\section{Acknowledgements}

The CLOtest slides were supplied by Delta-West Ltd, Perth, Western Australia.

5. Borsch, G., Adamek, R., Sandmann, M. et al. Comparison of biopsy urease test and histologic examination for detection of Campylobacter pylori in duodenal, antral and fundic biopsies. Hepato gastroenterol 1987, 34: 236-241. 